\title{
Measurement of the Actual Contact Area of Details by the Power of a Reflected Light Beam Directed to a Contact Surface Covered with a Film
}

\author{
Arkadiy Titov, Anna Sandulyak ${ }^{*}$, Mikhail Baharev, and Alexander Sandulyak \\ Moscow technological university, Moscow, RF
}

\begin{abstract}
Measurement of the actual area of details contact is important for control of friction and wear of such details. For this purpose, in particular, on a surface of one of details of couple covered with the thinnest layer of lubricant the thin film of coal is putted. On the raised dust surface impose the second detail of couple and squeeze them the application of normal loading. Light spots of contact are measured by planimetry. Instead of direct (labor-consuming) measurement of a large number of the areas of spots of contacts it is expedient to use integrated determination of the actual area of details - on the power of the beam of light reflected from ledges of a contact surface. The optical scheme of the device is developed, the efficiency of which for implementation is shown. Measurement of the actual area of details contact is important for control of friction and wear of such details. For this purpose, in particular, on a surface of one of details of couple covered with the thinnest layer of lubricant the thin film of coal is putted. On the raised dust surface impose the second detail of couple and squeeze them the application of normal loading. Light spots of contact are measured by planimetry. Instead of direct (labor-consuming) measurement of a large number of the areas of spots of contacts it is expedient to use integrated determination of the actual area of details - on the power of the beam of light reflected from ledges of a contact surface. The optical scheme of the device is developed, the efficiency of which for implementation is shown.
\end{abstract}

\section{Introduction}

At present, measuring the actual contact area of details are a very urgent task, since it largely determines the friction and wear of the details. Nowadays, the following methods for measuring the actual area of contact of surfaces are known: electrical; geometric; adhesive; optic; physicochemical [1]. Of the listed methods, the simplest and most convenient one should recognize adhesion, which is that a thin film of coal is deposited on the surface of one of the piece of a pair coated with a thin layer of lubricant, a second piece

*Corresponding author: anna.sandulyak@mail.ru 
of a pair is impressed to the evaporated surface and compressed by applying a normal load, light blots of touch measured by planimetration [2]. The disadvantage of the method is low productivity, since it is necessary to measure a large number of areas of contact spots. To eliminate this shortcoming, we proposed an integral method for determining the actual contact area of details by the power of the light beam reflected from the projections of the contact area [3].

\section{Calculations}

To implement the recommended method, we proposed a device whose schematic structure is shown in Fig. 1. The device contains: 1 - frequency generator, 2 - power amplifier, 3 - LED, a 4 - collimator lens, 5 - detail coated by film, 6 - lens, a 7 photodetector, 8 - amplifier, 9 - high frequency filter, 10 - computer. The device works as follows. From the generator 1 voltage through the amplifier 2 goes to the LED 3, the output of which produces an alternating luminous flux. LED 3 is in the focus of lens 4, forming a parallel light flux illuminating detail 5.

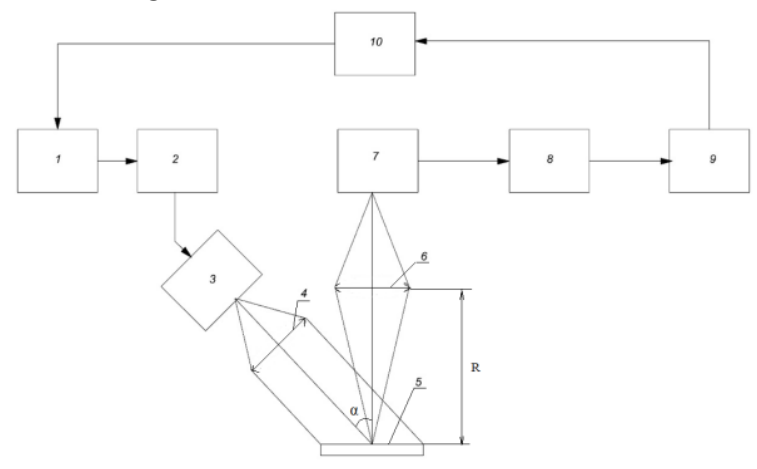

Fig.1. Schematic structure of the device for measuring the actual contact area of details

The light flux scattered from the surface of the detail 5 is collected by lens 6 on the photodetector 7, from the output of which the voltage through the amplifier 8 goes to the filter 9, which extinguishes the external illumination of the low frequency of $50 \mathrm{~Hz}$. Then the signal goes to the computer 10 , which processes the received information according to the developed algorithm, which will be considered later.

Define the basic characteristics of the device. To do this, it is necessary to determine the radiation power scattered from the surface of the detail 5. Suppose that the film and projections of part 5 reflect light according to Lambert's law (diffuse reflection). Then the power of the light beam reflected from the film entering the lens 6 , taking into account [3, 4], can be found from the expression:

$$
P_{1}=\frac{I S T_{n z} \tau D^{2} \cos \alpha}{4 R^{2}}
$$

where I - is the radiation intensity falling on the contact surface covered by the film; $\mathrm{S}$ - the area of the detail; $\mathrm{T}_{\text {пл }}$ - reflection coefficient of the film; $\tau$ - loss of light in optics; D diameter of lens $6 ; \mathrm{R}$ - the distance from the contact surface of detail 5 to the lens $6 ; \alpha$ angle between the normal to the contact surface and the direction to the lens 4 .

Then, the second detail is pressed against the contact surface of the first detail 5 and after deformation of the individual parts of the film, the second detail is removed. The contact surface is again illuminated with light. Then the radiation power reflected from the contact surface after deformation, taking into account [3], can be represented as: 


$$
P_{2}=\frac{I\left[S_{n l} T_{n l}+S_{6} T_{\partial}\right] \tau \mathrm{D}^{2} \cos \alpha}{4 R^{2}}
$$

where $\mathrm{S}_{6}$ - the area of the projections; $\mathrm{S}_{n л}$ - area of the detail occupied by the film; $\mathrm{T}_{\partial}$ - the reflection coefficient of the part corresponding to the reflection coefficient of the projections.

Taking into account that $\mathrm{S}=\mathrm{S}_{6}+\mathrm{S}_{n л}$ and expressions (1) and (2), we find the area of the projections:

$$
S_{6}=\left|\frac{4 R^{2} \mathrm{~S}_{n}\left(P_{1}-P_{2}\right)}{P D^{2} \cos \alpha\left(T_{n l}-T_{\partial}\right) \tau}\right|
$$

where $\mathrm{P}$ - power of the radiator; $\mathrm{S}_{n}$ is the area of the beam illuminating the part.

In order for the light flux from the entire area of the contact surface to completely reach the sensitive area of the photodetector, it is necessary to determine the parameters of the lens and the distance between it and the photodetector. To do this, we determine the lens magnification:

$$
\beta=-\frac{l_{\phi n}}{l_{\partial}}
$$

where $1_{\phi n}$ is the size of the photodetector; $1_{\partial}$ is the largest detail size. [4]:

The distance $\mathrm{R}$ can be expressed through the lens magnification and its focal distance $\mathrm{f}^{\prime}$

$$
R=\frac{(1-\beta)}{\beta} f^{\prime}
$$

The distance from the lens to the photodetector is:

$$
a=(1-\beta) f^{\prime}
$$

Taking into account (5), expression (3) takes the form:

$$
S_{b}=\left|\frac{4(1-\beta)^{2} \mathrm{f}^{\prime 2} \mathrm{~S}_{n}\left(P_{1}-P_{2}\right)}{P D^{2} \beta^{2} \cos \alpha\left(T_{n l}-T_{\partial}\right) \tau}\right|
$$

Let's find the sensitivity of the method, which is determined by the minimum area of projections that the device can detect. To do this, we use expression (7), in which the power difference $\mathrm{P} 1 \neg \mathrm{P} 2$ will correspond to the minimum power detected by the photodetector. Then using expression (7) taking into account [4] we obtain:

$$
S_{\text {s } \min }=\left|\frac{4(1-\beta)^{2} \mathrm{f}^{\prime 2} \mathrm{~S}_{n} \mathrm{gw} \sqrt{v}}{P D^{2} \beta^{2} \cos \alpha\left(T_{n l}-T_{\partial}\right) \tau}\right|
$$

where $\mathrm{g}$ is the threshold sensitivity of the photodetector $7, \mathrm{w}$ is the signal-to-noise ratio; $\mathrm{v}$ Bandwidth of the device.

We estimate the accuracy of the measurement. To this end, we select in the expression (7) parameters that have systematic errors, to which $\mathrm{S}_{n}, \alpha, \mathrm{T}_{n л}, \mathrm{~T}_{\partial}$, D can be assigned. These parameters can be measured when the instrument is adjusted. The remaining parameters included in expression (7) have random errors. The relative measurement error is found by differentiating expression (7) with respect to parameters that have random errors:

$$
\frac{\Delta S_{6}}{S_{6}}=\sqrt{4\left(\frac{\Delta \beta}{(1-\beta) \beta}\right)^{2}+4\left(\frac{\Delta f^{\prime}}{f^{\prime}}\right)^{2}+\left(\frac{\Delta P}{P}\right)^{2}}
$$

We estimate the parameters entering into (9). As shown in [4], the errors occurring in (9) 
are respectively equal to: $\frac{\Delta P}{P}=10^{-2}, \frac{\Delta f}{f}=10^{-4}, \frac{\Delta \beta}{\beta}=10^{-4}$.

Substituting the values of these parameters in (9), we obtain $\frac{\Delta S_{e}}{S_{e}} \approx 1 \%$.

By expression (8), we determine the sensitivity of the device. For the experimental sample, elements with the following parameters were chosen. The source of radiation is a white LED with a power of $50 \mathrm{Lm}$. The photodetector has a sensitive area of $3 \times 3 \mathrm{~mm}$ with a threshold sensitivity of $2.73 * 10^{-11} \mathrm{Lm} / \mathrm{Hz}^{0.5}$. The condenser forms a beam of $21 \mathrm{~mm}$ in diameter on the investigated surface. The magnification of the lens 6 can be found from the expression (4) taking into account the size of the photodetector and beam diameter $\beta=$ 0.142 . The lens has a diameter of $20 \mathrm{~mm}$ and a focal length of $20 \mathrm{~mm}$. The angle $\alpha=56^{\circ}$. The modulation frequency is chosen to be $5 \mathrm{kHz}$, and the signal-to-noise ratio $\mathrm{w}=10$. Coefficient of reflection from the black film $\mathrm{T}_{n \pi}=0.04$, and from the detail $\mathrm{T}_{\partial}=0.8$. Loss in optics $\tau=0$.7. Substituting the above parameters in (8), we obtain $\mathrm{S}_{\text {6min }} \approx 70 \mu \mathrm{m}^{2}$. When the emitter power is increased (using a laser), the sensitivity can be significantly increased.

Consider now how information is processed by a computer 10. As a medium for measuring the output voltage and generating a modulating one, we select the Lab View virtual programming environment and as a hardware part for receiving and transmitting data, a sound card.

Generation of the modulating signal is effected by the linear audio output of the computer sound card 10. The procedure for generating this signal passes in two stages. On the first stage, a sinusoidal signal is generated by a component representing itself as a virtual signal generator Simulate Signal. Figure 2 shows that the frequency is set to $5 \mathrm{kHz}$.

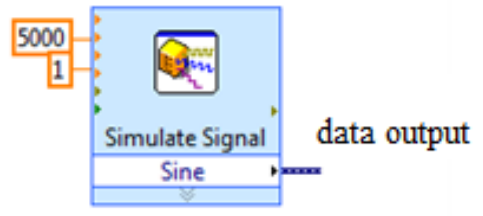

Fig.2. Virtual Generator

At the second stage, the signal is output via the line port of computer 10 via an element like Play Waveform. In this element, you can specify only the device number that will reproduce the signal.

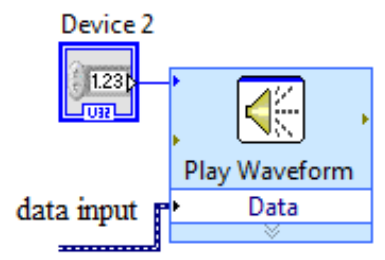

Fig.3. Element for signal output to the line output of the computer's audio card

The signal from the output of the filter 9 goes to the line input of the computer's audio card 10 and is entered into the program using the standard component Acquuire Sound, which accesses its audio card. 


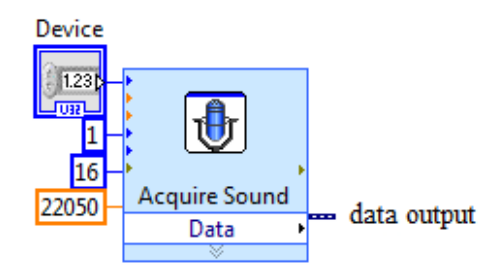

Fig.4. Data access element of the input line port

As you can see in Figure 4, on this component can be relocate device number (Device ID), sampling data per channel (Number of Samples/ch), sampling rate (95 kHz), bit data (16 bits) and the number of channels involved ( 1 channel).

Then the signal goes to the amplitude meter - the standard component Tone Measurements Express VI. Lab View. It determines the amplitude of the input signal. The tasks that this component solves, are the search for a single signal with the largest amplitude, or search for a specified frequency range to search for a single signal with the largest amplitude.

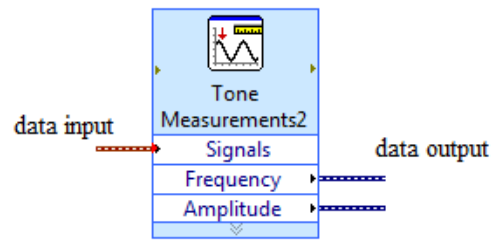

Fig.5. Tone Measurements element for analyzing the sinusoidal signal

To perform amplitude measurements, it is necessary to make a reference system. This function allows you to start measuring at any value of the initial signal. Therefore, the following circuit was shown, shown in Figure 6, performing the position setting to zero.

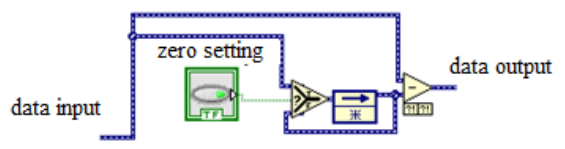

Fig.6. Link to set the origin to zero

Here, Lab View, by pressing a button, captures data from the bus coming from the amplitude analyzer and stores these values in the Feedback Node component (remembers the last value), and after disabling the button, the data is stored and subtracted from the signal coming from the amplitude analyzer. The overall system of program is shown in Fig. 7.

After the development and fabrication of the device model, experimental studies were carried out. To do this, samples were made with different contact areas.

These samples were in turn installed in the device instead of detail 5 (Fig. 1). Using computer 10 , the dependence of the voltage of the signal on different contact areas was obtained. The graph of this relationship is shown in Fig. 8. 


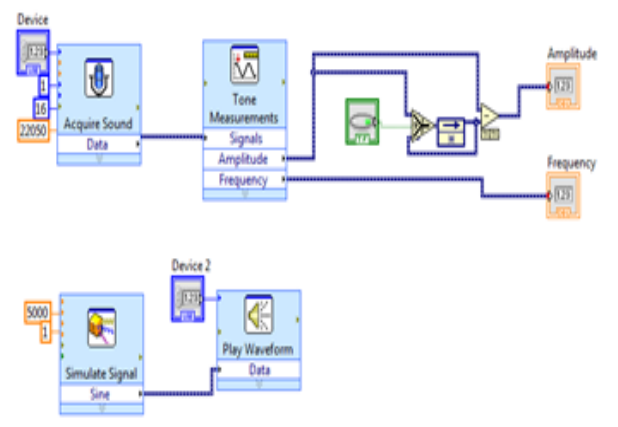

Fig.7. Program construction for the contact area meter

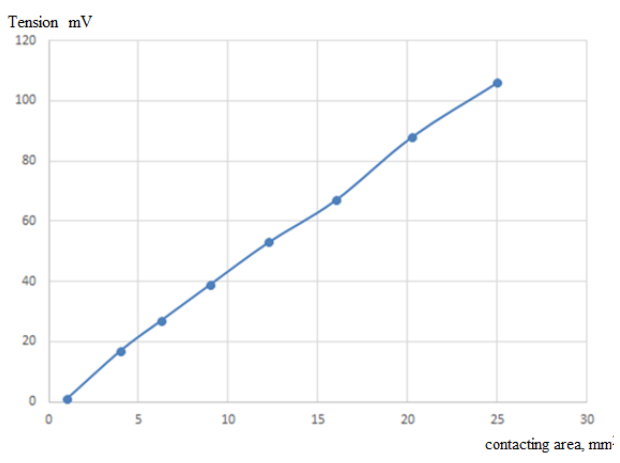

Fig.8. Voltage versus contact area

\section{Conclusions}

As you can see from Figure 8, the graph has a fairly linear character, which corresponds to the theoretical positions.

Thus, analytical expressions for calculating the basic parameters of the device were obtained in this paper, its sensitivity and measurement accuracy were determined, a schematic structure of the device and a special program for the computer were developed, a model was developed and the actual contact area of the details was measured.

\section{Acknowledgements}

The research is conducted with financial support from RFFI within the frameworks of research project № 16-38-60034 mol_a_dk and the support of the Ministry of Education and Science of the Russian Federation for the state task in the field of scientific activities no. 9.9626.2017.

\section{References}

1. V.M Gryazev. Izvestiya TulGU. Technical Science 10, 26-32 (2013)

2. I.I. Berkovich, D.G. Gromakovskiy. Tribology. Physical bases, mechanics and technical applications: The textbook for higher education institutions. Samara (2000) p. 213.

3. Patent RU 2642915. Way of measurement of the actual area of contact of details. By Albagachiev A.Y., Titov A.A., Sandulyak A.A. (2018).

4. A.A. Titov. Measurement techniques. 56 (6), 628-650 (2013). 